\title{
Pulmonary delivery of siRNA as a novel treatment for lung diseases
}

\author{
Rima Kandil ${ }^{1}$ \& Olivia M Merkel*,1 \\ ${ }^{1}$ Department of Pharmacy, Pharmaceutical Technology \& Biopharmaceutics, Ludwig-Maximilians University, Butenandtstraße 5-13, \\ 81337 Munich, Germany \\ *Author for correspondence: Tel.: +49 892180 77025; Fax: +49 892180 77020; Olivia.merkel@Imu.de
}
${ }^{6} \mathrm{~A}$ crucial step for the transfer of pulmonary siRNA delivery from bench to bedside is to develop and characterize inhalable drug products."

\section{Graphical abstract:}

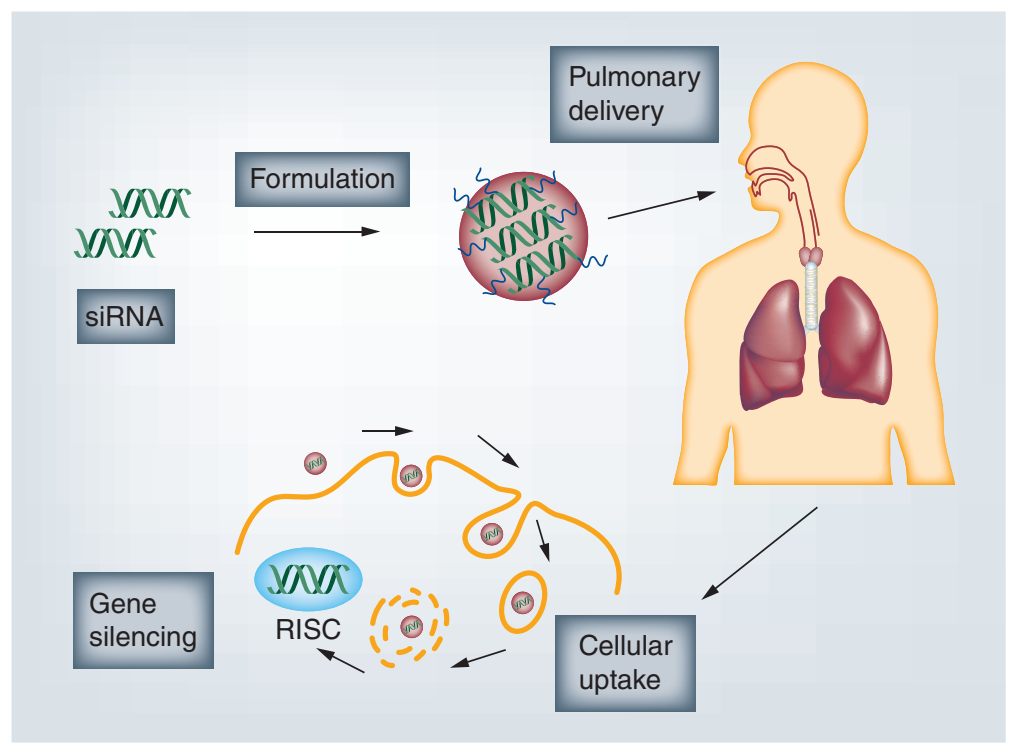

First draft submitted: 19 February 2019; Accepted for publication: 12 March 2019; Published online: 17 April 2019

Keywords: inhalation • lung diseases • pulmonary delivery • RNAi • siRNA

The lung offers various beneficial characteristics as a target organ for therapeutic approaches. Beside the large (hundreds of square meters) and very well perfused $(5 \mathrm{l} / \mathrm{min})$ surface area, extremely thin epithelium $(0.1-0.2 \mu \mathrm{m})$ and high blood volume in pulmonary capillaries $(0.25 \mathrm{l})$, it also captivates with a relatively low enzyme activity and slow surface clearance [1]. Owing to its location and function, the pulmonary region is susceptible to a number of specific diseases, being directly accessible to harmful substances. Lung-related disorders therefore contribute substantially to the global disease burden in regards to public health and economic resources. For a considerable number of those pathologies including asthma, idiopathic pulmonary fibrosis, respiratory syncytial virus and, moreover, lung cancer as the leading cause of cancer death worldwide, the underlying cause is elevated transcription of certain genes $[2,3]$. Therefore, the interference with gene expression via RNA interference in principle offers great potential to help address unmet medical needs in this regard.

Since small interfering RNA (siRNA) is the most extensively studied RNAi molecule for therapeutic application, accounting for half of all recent clinical studies focusing on this mechanism [4], direct delivery of siRNA to diseased lung tissue presents a promising treatment option. The siRNA can induce post-transcriptional gene silencing by 
inhibition of respective mRNA, making it possible to treat even diseases for which currently no drugs are available [5]. Compared with other gene therapy approaches, siRNA provides several benefits such as robustness of expression, specificity of inhibitory effect, high efficiency even at low concentrations and reduced toxicity [1]. Furthermore, unlike DNA, siRNA does not need to be delivered to the cell nucleus, but only to the cytoplasm in order to bring about its therapeutic effect. Systemic injection of siRNA has proven to be difficult as it is easily degraded by ubiquitous nucleases and rapidly excreted via the kidneys, not being retained by glomerular filtration [6]. Local delivery, however, enables direct transport to the target site while circumventing the first-pass effect resulting in lower doses and respective side effects.

The primary barrier for pulmonary siRNA delivery is the extensively branched structure of the airways with variable lengths and diameters, followed by the presence of lung fluids, such as mucus and surfactant [7], as well as mucociliary clearance mechanisms including alveolar macrophages. As the human respiratory tract occupies an efficient system for gas exchange and protection against foreign particles, careful consideration of these natural defense mechanisms is necessary when formulating pulmonary therapeutics [8]. To improve siRNA stability and efficacy without using delivery systems, it can be chemically modified or conjugated to appropriate biomolecules [9]. Beside this, carrier vectors can of course help to overcome delivery challenges of naked nucleic acids. Due to improved toxicity profiles concerning immunogenicity and tumorigenicity as well as ease and reproducibility of manufacturing, nonviral vectors are mostly preferred over virus-based systems [10]. A great range of different materials is available, including polymers, lipids, peptides and inorganic material, which can furthermore be tailored with diverse surface modifications [11], such as targeting ligands or membrane-active substances.

Administration of siRNA to the lungs is usually performed via inhalation or intranasally, as both ways present easy and noninvasive options. For inhalation, siRNA can be formulated into liquid aerosols produced by inhalers or nebulizers or as a dry powder aerosol [9]. Currently available inhalation devices comprise pressurized dry powder inhalers (DPIs), nebulizers, soft mist inhalers and the most commonly used metered dose inhalers (pMDIs). For successful pulmonary application, particle deposition near the targeted lung cells is fundamental, arrangeable with optimal particle size, shape and density. The ideal aerodynamic diameter of droplets or powder particles would be between 1 and $5 \mu \mathrm{m}$, as smaller particles are very likely to be exhaled, while larger ones might easily deposit in the throat and upper airway walls [7]. By precisely controlling these particle characteristics and thereby deposition mechanics of drug formulations, specific regions in the pulmonary tract can be reached [12]. Even more distinct delivery is possible by targeting of certain cell types in the lung, for example by coupling ligands that bind to receptors expressed on the surface of target cells. The native glycoprotein transferrin, for instance, was shown to be an effective mediator to reach activated $T$ cells as disease mediators in asthmatic lungs [13] or malignant lung cancer cells [14]. After successfully reaching the target cells, siRNA particles have to be efficiently taken up which is mostly achieved by endocytosis. It is then crucial for the nucleic acid to escape the endosomes to avoid degradation upon acidification and to achieve cytoplasmic delivery to the RNA-induced silencing complex, the target location of siRNA. In fact, recent studies identified escape of the endosomal pathway as the rate-determining step in delivery of therapeutic molecules [15], justifying the various approaches to improve endosomal release. Among others, membrane fusion or disruption, pore formation and osmotic lysis via buffering effects are currently investigated options [16].

Almost all recent studies investigating the pulmonary delivery of therapeutic siRNA showed positive outcomes, prooving a working principle. Nevertheless, some reoccurring challenges were identified along the way, including distribution of siRNA in tissues other than the lung, safety issues of delivery vectors, unclear effects of longterm application and a poor in vitro-in vivo correlation [2]. A crucial step for the transfer of pulmonary siRNA delivery from bench to bedside is to develop and characterize inhalable drug products. Appropriate ways to analyze aerosolized siRNA delivery vectors in vitro comprise dissolution tests, inertial cascade impaction, delivered dose uniformity assay, laser diffraction and laser Doppler velocimetry, as well as the isolated lung perfusion model as a useful ex vivo option. Subsequently, formulations can be examined in vivo using methods such as $\gamma$-scintigraphy, 3D SPECT or MRI fluorescence imaging [8].

The majority of in vivo studies are still carried out in mice, as they are small, rather economical in terms of husbandry and offer fast and efficient reproduction. However, rodent airways distinctly differ from those of humans in regards to anatomy, histology and physiology [17]. As different pharmacokinetic properties such as particle deposition and mucociliary clearance strongly affect the eventual active dose, it is essential to consider this fact before extrapolating these results to human application. Furthermore, animal models should ideally mimic the clinical conditions as accurately as possible to examine potential medications in a realistic disease environment 
and integrating the fact that respiratory function is compromised in patients with lung diseases. Due to the ease of experimental setup and control, the application route of choice is usually intratracheal or intranasal. Although intranasal delivery is very straightforward, since the formulation just has to be instilled in the nasal cavity and breathed in, humans are not obligate nose breathers and their nasal cavity filters out the vast amount of particles, resulting in a low lung deposition [18]. Intratracheal administration, on the other hand, does not perfectly translate inhalation outcomes, resulting in comparably less uniform particle deposition and neglecting of oropharynx deposition, eventuating in a lower total drug loss [8]. Moreover, this method is not suitable for human clinical studies or even usage in the clinical routine due to its invasiveness, as inserting a tube within a surgical incision is highly impracticable and uncomfortable for the patient [19]. Accordingly, focusing more on the testing of actual inhalable dosage forms would be rather expedient. Most studies also use liquid formulations, raising problems with regard to stability, as those are highly susceptible to nuclease contamination and not suitable for long-term storage. Therefore, dry powder formulations seem to be the more promising option and have, not surprisingly, gained more and more attention in recent work [2].

Altogether, the therapeutic potential of RNAi in lung diseases was clearly demonstrated in several in vitro studies as well as in clinical trials. With patisiran $\left(\mathrm{ONPATTRO}^{\mathrm{TM}}\right)$ recently being approved in the USA and the EU as the very first siRNA medication [20], significant progress in the development of RNAi-based therapeutics has been made, paving the way for more products to follow. As a large share of airway-related diseases evolve around inflammatory processes for which long-term therapies are needed, it is inevitable to further investigate long-time stability of siRNA formulations in appropriate devices as well as effects of continuous repeated application. Here, both the nucleic acid itself, with particular regards to undesired immune responses and potential off-target effects, and the respective carrier system have to be closely examined. Preferably, efforts should be further focused on the development of biodegradable delivery vectors in order to achieve safe and compatible formulations. With all points considered, the pulmonary route presents a promising approach for siRNA-based therapies presumable to be realizable in the near future.

\section{Acknowledgments}

The authors are grateful to A Mehta for diligent proofreading of this article.

\section{Financial \& competing interests disclosure}

The authors are funded by ERC-2014-StG - 637830. The authors have no other relevant affiliations or financial involvement with any organization or entity with a financial interest in or financial conflict with the subject matter or materials discussed in the manuscript apart from those disclosed.

No writing assistance was utilized in the production of this manuscript.

\section{References}

1. Ray A, Mandal A, Mitra AK. Recent patents in pulmonary delivery of macromolecules. Recent Pat. Drug Deliv. Formul. 9(3), 225-236 (2015).

2. Ruigrok MJR, Frijlink HW, Hinrichs WLJ. Pulmonary administration of small interfering RNA: the route to go? J. Control. Rel. 235, 14-23 (2016).

3. Bray F, Ferlay J, Soerjomataram I, Siegel RL, Torre LA, Jemal A. Global cancer statistics 2018: GLOBOCAN estimates of incidence and mortality worldwide for 36 cancers in 185 countries. CA Cancer J. Clin. 68(6), 394-424 (2018).

4. Ozcan G, Ozpolat B, Coleman RL, Sood AK, Lopez-Berestein G. Preclinical and clinical development of siRNA-based therapeutics. Adv. Drug Deliv. Rev. 87, 108-119 (2015).

5. Merkel OM, Kissel T. Nonviral pulmonary delivery of siRNA. Acc. Chem. Res. 45(7), 961-970 (2012).

6. Tatiparti K, Sau S, Kashaw SK, Iyer AK. siRNA delivery strategies: a comprehensive review of recent developments. Nanomaterials (Basel) 7(4), e77 (2017).

7. Qiu Y, Lam JK, Leung SW, Liang W. Delivery of RNAi therapeutics to the airways - from bench to bedside. Molecules 21(9), E1249 (2016).

8. Youngren-Ortiz SR, Gandhi NS, Espana-Serrano L, Chougule MB. Aerosol delivery of siRNA to the lungs. Part 1: rationale for gene delivery systems. Kona 33, 63-85 (2016).

9. Chen J, Tang Y, Liu Y, Dou Y. Nucleic acid-based therapeutics for pulmonary diseases. AAPS PharmSciTech 19(8), 3670-3680 (2018).

10. Merkel OM, Rubinstein I, Kissel T. siRNA delivery to the lung: what's new? Adv. Drug Deliv. Rev. 75, 112-128 (2014).

11. Kandil R, Merkel M. Therapeutic delivery of RNA effectors: diseases affecting the respiratory system. Pharmazie 71(1), 21-26 (2016). 
12. Moschos SA, Usher L, Lindsay MA. Clinical potential of oligonucleotide-based therapeutics in the respiratory system. Pharmacol. Ther. 169, 83-103 (2017).

13. Xie Y, Kim NH, Nadithe $\mathrm{V}$ et al. Targeted delivery of siRNA to activated $\mathrm{T}$ cells via transferrin-polyethylenimine (Tf-PEI) as a potential therapy of asthma. J. Control. Rel. 229, 120-129 (2016).

14. Pooja D, Kulhari H, Tunki L et al. Nanomedicines for targeted delivery of etoposide to non-small cell lung cancer using transferrin functionalized nanoparticles. RSC Advances 5(61), 49122-49131 (2015).

15. Smith SA, Selby LI, Johnston APR, Such GK. The endosomal escape of nanoparticles: towards more efficient cellular delivery. Bioconjug. Chem. 30(2), 263-272 (2018).

16. Selby LI, Cortez-Jugo CM, Such GK, Johnston APR. Nanoescapology: progress toward understanding the endosomal escape of polymeric nanoparticles. Wiley Interdiscip. Rev. Nanomed.Nanobiotechnol. 9(5), (2017). https://doi.org/10.1002/wnan.1452

17. Kolanjiyil AV, Kleinstreuer C, Kleinstreuer NC, Pham W, Sadikot RT. Mice-to-men comparison of inhaled drug-aerosol deposition and clearance. Respir. Physiol. Neurobiol. 260, 82-94 (2019).

18. Hickey AJ, Garcia-Contreras L. Immunological and toxicological implications of short-term studies in animals of pharmaceutical aerosol delivery to the lungs: relevance to humans. Crit. Rev. Ther. Drug Carrier Syst. 18(4), 387-431 (2001).

19. Lam JK, Liang W, Chan HK. Pulmonary delivery of therapeutic siRNA. Adv. Drug Deliv. Rev. 64(1), 1-15 (2012).

20. Hoy SM. Patisiran: first global approval. Drugs 78(15), 1625-1631 (2018). 